\title{
Dislocation Substructure Evolution during Hydrostatic Extrusion of Al-Mg-Si Alloy
}

\author{
W. Chrominski* And M. LeWAndowska \\ Warsaw University of Technology, Faculty of Materials Science and Engineering, \\ Wołoska 141, 02-507 Warsaw, Poland
}

\begin{abstract}
Hydrostatic extrusion is a technique which allows to produce rods with ultrafine grains and unexpectedly enhanced mechanical properties caused by grain refinement. However, the mechanism of such a refinement is not fully understood at this stage. 6xxx aluminium alloys series are usually processed by extrusion. In this study, commercial 6082 aluminium alloy was extruded at ambient temperature in a cooled die in two stages to the true strain of $\varepsilon=3.2$. Such a processing results in a not fully refined microstructure which allows to study different stages of grain refinement. The texture, dislocation substructures and grain refinement were investigated using electron backscatter diffraction and transmission electron microscopy techniques. The results revealed that two main texture components are present in the extruded rods - $\langle 111\rangle$ fiber texture and $\langle 001\rangle$ recrystallized grains. Transmission electron microscopy inspection revealed dislocation structures that can be associated with different stages of plastic deformation according to the low energy dislocation structures hypothesis proposed by Kuhlmann-Wilsdorf.
\end{abstract}

DOI: $10.12693 /$ APhysPolA.128.585

PACS: 81.05.Bx, 61.72.Ff, 81.20.Hy, 81.40.Ef, 81.40.Lm

\section{Introduction}

Plastic deformation of metals usually results in a substantial increase in dislocation density which arrange themselves into different structures, in particular low energy ones. The structure formed depends primarily on the imposed strain, crystal lattice and stacking fault energy (SFE). In the case of $\mathrm{Al}$ (high SFE), the size of dislocation cells decreases with increasing strain [1, 2]. At sufficiently high strain, they rotate around a common axis and form new high angle boundaries [3,4] which also results in a sharp texture [5-8]. As a consequence of such a structural evolution, the grain can decrease below $1 \mu \mathrm{m}$.

Ultrafine grained (UFG) materials are attracting a growing attention due to their superb mechanical properties [5, 9-15]. This recognition resulted in the development of their fabrication methods, in particular severe plastic deformation (SPD) techniques, in which grain refinement occurs as a consequence of extremely high strains [5]. However, it should be noted that grain size reduction to UFG regime in metals can also be achieved by means of conventional deformation techniques, such as rolling [16]. The microstructure evolution as a function of imposed strain was well described for rolling [17] and equal channel angular pressing (ECAP) [4]. The aim of this work is to discuss microstructural evolution during hydrostatic extrusion (HE) which has been already proven to be an efficient method of grain size refinement in a number of metals and alloys, including technically pure aluminium $[15,19]$. It has been shown previously that for technically pure aluminium, a true strain

* corresponding author; e-mail: wichr@inmat.pw.edu.pl of $\varepsilon=2.77$ is sufficient to obtain refined microstructure [19]. However, it should be noted here that pure metals are susceptible to recrystallize dynamically during plastic deformation.

\section{Material and methods}

A commercial $6082 \mathrm{Al}-\mathrm{Mg}-\mathrm{Si}$ alloy was used in this study. The material was supplied in a rod form with a diameter of $50 \mathrm{~mm}$ in $\mathrm{T} 6$ hardened condition. The samples were solution annealed at $520^{\circ} \mathrm{C}$ for $2 \mathrm{~h}$ and quenched into water in order to obtain supersaturated solid solution. Such a condition allows cold plastic deformation. HE was performed at ambient temperature. The unwanted warming of the specimen was reduced by a cooling system. Two stage extrusion resulted in an accumulative strain of $\varepsilon=1.8$ after first pass and $\varepsilon=3.2$ after the second. The final product was in a form of a rod having $10 \mathrm{~mm}$ in diameter.

Samples for electron backscattered diffraction (EBSD) and transmission electron microscopy (TEM) inspection were in the form of thin discs of cross-sections taken from the center of the extruded rod with spark erosion machine and wire saw. Surface of the specimens for EBSD analysis was prepared electrochemically with final ion polishing. Electron transparent regions for TEM observations were obtained by electrochemical thinning.

EBSD measurements were performed on a $\mathrm{Hi}^{-}$ tachi SU70 scanning electron microscope (operation voltage of $20 \mathrm{kV}$ ) with the Schottky emitter. The data for texture analysis were taken with $0.5 \mu \mathrm{m}$ step which resulted in orientation maps with dimensions of $100 \times$ $100 \mu^{2}$. Results were used to built inverse pole figures and calculate volume fraction of each of the texture component as a ratio of the area related to a particular orientation to the whole examined region. $0.5 \mu \mathrm{m}$ 
step is too coarse for providing a detailed microstructure analysis of severely deformed aluminum so discussion of orientation maps is omitted in this study.

TEM observations were performed on JEOL JEM1200 EX II microscope with accelerating voltage of $120 \mathrm{kV}$ and high double tilt range for setting desired diffraction conditions. TEM investigations were focused on the dislocation structure dependence on the grain orientation relatively to extrusion direction. General observations were performed with bright field mode. Dislocation structures were investigated in terms of number of different Burgers vectors $\boldsymbol{b}$ (determined with invisibility criterion $\boldsymbol{g} \cdot \boldsymbol{b}=0$ ) participated in a specific dislocation wall. Images of dislocation structures were recorded using $\boldsymbol{g}-\mathbf{3} \boldsymbol{g}$ weak beam dark field imaging condition with slightly positive deviation parameter $s$ for sharp images of dislocation lines.

\section{Results and discussion}

The first HE pass of supersaturated 6082 aluminium alloy results in sharp double texture with $\langle 001\rangle$ and $\langle 111\rangle$ components parallel to extrusion direction (Fig. 1). The texture components fractions equals to $22 \%$ and $78 \%$ for $\langle 001\rangle$ and $\langle 111\rangle$, respectively. After the second pass, the fraction of $\langle 001\rangle$ component did not change significantly.

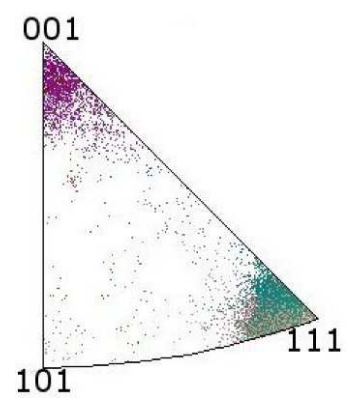

Fig. 1. Inverse pole figure taken from 6082 aluminium alloy hydrostatically extruded to the true strain of $\varepsilon=$ 1.8 shows sharp double texture.

According to literature, deformation substructure strongly depends on orientation [20], i.e. in $\langle 111\rangle$ grains dense dislocations walls form whereas in $\langle 001\rangle$ grains stable cell dislocation structure exists. This implies significant differences in differently oriented grains in HE processed sample. To provide a better insight into structural varieties, TEM study was performed.

A bright field image of dislocations configuration in grain with $\langle 001\rangle$ parallel to extrusion direction (orientation confirmed with diffraction pattern presented as inset) after the first HE pass is presented in Fig. 2a. One can see dislocation tangles which can be considered as early stages of dislocation cell structure formation. One should also note differences in diffraction contrast between the cells ${ }^{\varsigma}$ interior and the regions with dislocations
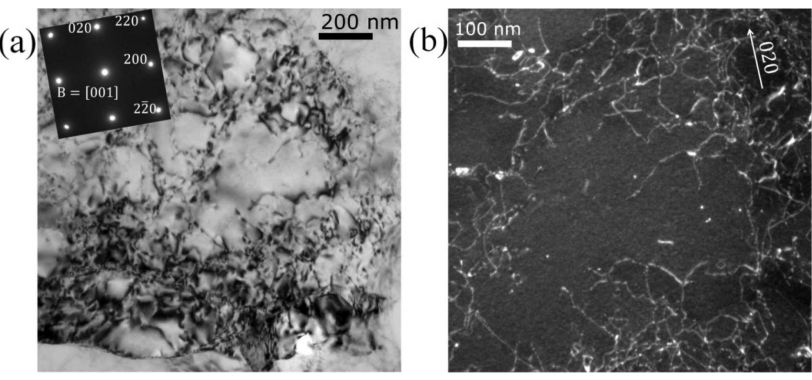

Fig. 2. Images of dislocation structure in grains with $\langle 001\rangle$ parallel to extrusion direction in 6082 aluminium alloy, $\varepsilon=1.8$ : (a) bright field image showing dislocation arrangement typical for early stages of plastic deformation (b) weak beam dark field image with excited diffraction vector $\boldsymbol{g}=[020]$.

tangles at the bottom of image. The former are characterized with relatively uniform contrast (see area in the center of Fig. 2a), while the latter features significant changes in contrast (bottom of Fig. 2a). Cells, which are visible in Fig. 2b, are the very early stage of low energy dislocation structure (LEDS) formation by dislocations with different Burgers vectors (confirmed by diffraction contrast experiment). The basic feature of LEDS is that the range of the stress field does not exceed the wall due to the superposition of stress fields arising from dislocation cores with different Burgers vectors $[1,2]$. The dislocation structure visible on the bottom of Fig. 2a is not ordered in energy lowering structures although it also contains dislocations with different Burgers vectors. As a result the local fluctuations of the Bragg diffraction condition diffraction contrast inhomogeneities can be observed. (a)

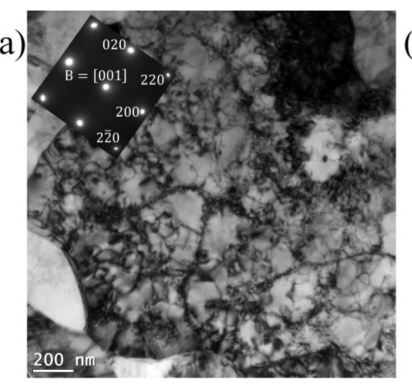

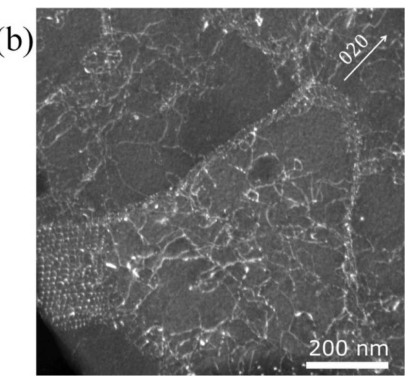

Fig. 3. Images of dislocation structure in with $\langle 001\rangle$ parallel to extrusion direction in 6082 aluminium $\varepsilon=3.2$

(a) bright field image showing dense dislocation walls

(b) weak beam dark field image with excited diffrac-

tion vector $\boldsymbol{g}=[020]$ of dislocation subgrain with single

Burgers vector dislocations arrangement inside.

Further plastic deformation, to a true strain of 3.2 , brings about the evolution of dislocation cells which become more developed as presented in Fig. 3a. Mean equivalent diameter of such cells is $360 \mathrm{~nm}$. Besides well developed LEDS, some dislocations can be noticed within cells as presented in Fig. 3b. Tilting to excite different diffraction vectors $\boldsymbol{g}$ showed that invisibility criterion was 
satisfied for $\boldsymbol{g}=[2-20]$ which means that they are a single $\boldsymbol{b}=[-110]$ structure which according to LEDS hypothesis is not stable.

The formation of such a microstructure can be elucidated as follows. Plastic deformation results in the generation of dislocations which rearrange themselves into LEDS, which are known to be stable. One of the examples of LEDS are dislocation cells which separate grain domains in which different slip systems operate. According to the model of structure development in heavily worked materials dislocation cells can accommodate plastic deformation until they reach critical dimensions in the fifth stage of work hardening [21]. In our case, the accumulated deformation is relatively low and one can conclude that the dislocation cells presented in Fig. 3 can still accommodate plastic deformation and evolve into finer ones.

(a)

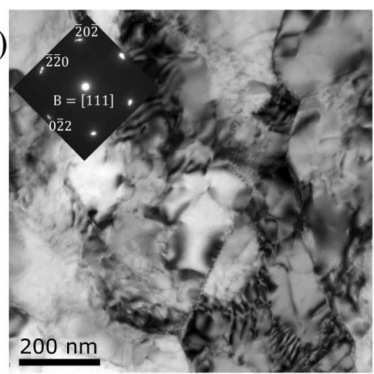

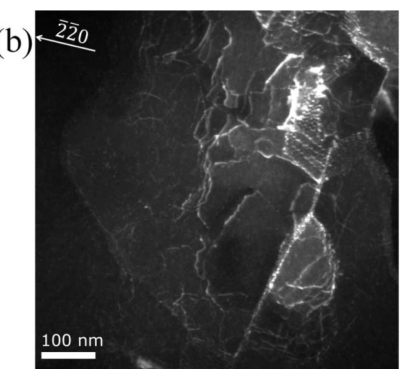

Fig. 4. Images of dislocation structure in fiber grains with $\langle 111\rangle$ parallel to extrusion direction in 6082 aluminium alloy $\varepsilon=1.8$ (a) bright field image of $\langle 111\rangle$ oriented region, note non-homogenous amplitude contrast (b) weak beam dark field image of dislocation structure within considered region.

The regions with $\langle 111\rangle$ parallel to extrusion direction differ significantly from $\langle 001\rangle$ ones discussed previously. Figure 4a presents a typical view of such a region. First characteristic feature is heterogeneous amplitude contrast within the observed area which made it difficult to determine any microstructure element with bright field imaging. Weak beam image of the same area presented in Fig. 4b reveals dislocation walls and some individual dislocations between them. Contrast on the left side of the image becomes weaker in comparison to the right side because of the residual stresses that cause bending of the lattice planes. Weak beam imaging is very sensitive to the deviation parameter $s$ that strongly depends on diffraction conditions. Even small deflection from perfect imaging conditions results in contrast weakening. For comparison, Fig. $2 \mathrm{~b}$ shows the area with the same magnitude. The contrast is uniform on whole image because of no residual stresses are present in this region. This observation implies that observed structures in $\langle 111\rangle$ grains are not LEDS. During diffraction contrast experiment dislocation invisibility criterion was satisfied for all dislocations within cell when $\boldsymbol{g}=[202]$. Furthermore, microstructure in $\langle 111\rangle$ oriented grains is much more developed than in $\langle 001\rangle$ grains (at strain level of $\varepsilon=1.8$ ) as the former possess relatively well developed cell structure with an average size of about $300 \mathrm{~nm}$, whereas in the latter cells are only about to being formed.

Figure 5 shows well developed, fine grains with $\langle 111\rangle$ parallel to extrusion direction typical for the sample deformed to larger strain. Diffraction pattern presented as an inset in the figure is a ring related with (220) planes which are in $\boldsymbol{B}=[111]$ zone axis. The mean size of grains equals to $240 \mathrm{~nm}$ and it is slightly smaller than those measured for dislocation cells in the previous HE stage in similarly oriented region. This indicates that cells size shrinks together with progression of plastic deformation before rotations. As it was already mentioned above, the diffraction pattern is now a ring which confirms increment of misorientation angles between cells which transform into well developed grains as can be seen in Fig. 5 . This microstructure type is the most advanced stage of grains development found in the investigated material.

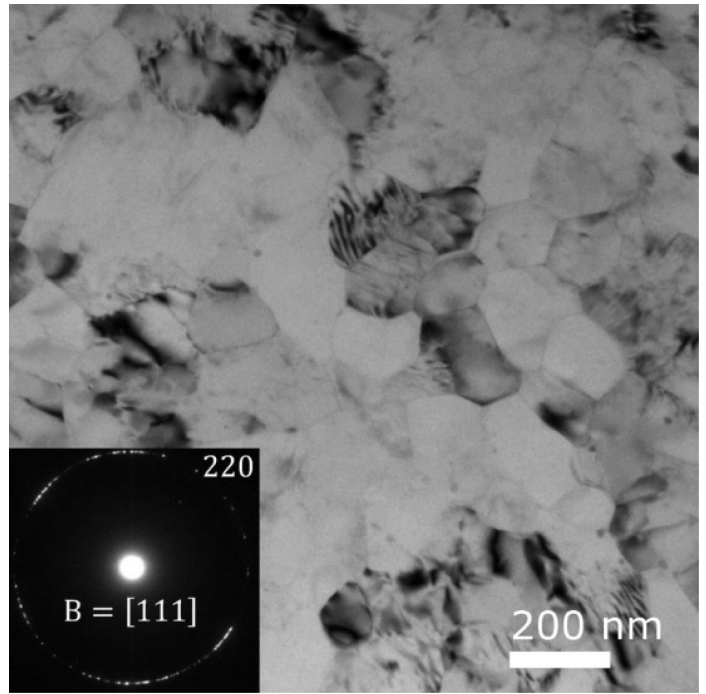

Fig. 5. Image of microstructures in fiber grains with $\langle 111\rangle$ parallel to extrusion direction in 6082 aluminium alloy $\varepsilon=3.2$.

\section{Conclusions}

Hydrostatic extrusion of a single phase (supersaturated solid solution) 6082 aluminium alloy results in double fiber textured grains with various dislocation substructures. $\langle 001\rangle$ oriented grains contain stable cellular structure which evolves as plastic deformation continues. $\langle 111\rangle$ grains show more advanced structures which result in refined, well developed grains separated with high angle grain boundaries.

\section{Acknowledgments}

This work was carried out within a NANOMET Project financed under the European Funds for Regional Development (Contract no. POIG.01.03.01-00-015/08). 
Authors would like to thank Dr. W. Pachla and Dr. M. Kulczyk from Institute of High Pressure Physics of the Polish Academy of Sciences for performing hydrostatic extrusion experiment.

\section{References}

[1] D. Kuhlmann-Wilsdorf, Mater. Sci. Eng. A 113, 1 (1989).

[2] D. Kuhlmann-Wilsdorf, Mater. Sci. Eng. A 86, 53 (1987).

[3] P.B. Prangnell, J.R. Bowen, P.J. Apps, Mater. Sci. Eng. A 375-377, 178 (2004).

[4] M. Cabibbo, W. Blum, E. Evangelista, M.E. Kassner, M.A. Meyers, Metall. Mater. Trans. A 39, 181 (2008).

[5] R.Z. Valiev, R.K. Ismagaliev, I.V. Alexandrov, Prog. Mater. Sci. 45, 103 (2000).

[6] H. Asgharzadeh, A. Simichi, H.S. Kim, Mater. Sci. Eng. A 528, 3981 (2011).

[7] I.J. Beyerlein, L.S. Toth, Prog. Mater. Sci. 54, 427 (2009).

[8] J.J. Sidor, R.H. Petrov, L.A.I. Kestens, Mater. Charact. 62, 228 (2011).

[9] R.Z. Valiev, Y. Estrin, Z. Horita, T.G. Langdon, M.J. Zehetbauer, Y.T. Zhu, JOM 58, 33 (2006).
[10] M.A. Meyers, A. Mishra, D.J. Benson, Prog. Mater. Sci. 51, 427 (2006).

[11] J.K. Kim, H.K. Kim, J.W. Park, W.J. Kim, Scr. Mater. 53, 1207 (2005).

[12] B. Adamczyk-Cieslak, J. Mizera, K.J. Kurzydlowski, Mater. Charact. 62, 327 (2011).

[13] H.J. Roven, H. Nesboe, J.C. Werenskiold, T. Seibert, Mater. Sci. Eng. A 410-411, 426 (2005).

[14] W. Blum, X.H. Zeng, Acta Mater. 57, 1966 (2009).

[15] M. Lewandowska, K.J. Kurzydlowski, J. Mater. Sci. 43, 7299 (2008).

[16] Y. Saito, H. Utsunomiya, N. Tsuji, T. Sakai, Acta Mater. 47, 579 (1999).

[17] B. Bay, N. Hansen, D.A. Hughes, D. KuhlmannWilsdorf, Acta Metall. Mater. 40, 205 (1992).

[18] A.P. Zhilyaev, G.V. Nurislamova, B.K. Kim, M.D. Baro, J.A. Szpunar, T.G. Langdon, Acta Mater. 51, 753 (2003).

[19] M. Lewandowska, Solid State Phenom. 114, 109 (2006).

[20] X. Huang, G. Winther, Philos. Mag. 87, 5189 (2007).

[21] M. Zehetbauer, V. Seumer, Acta Metall. Mater. 41, 577 (1993). 